\title{
Towards More Capable and Less Invasive Robotic Surgery in Orthopaedics
}

\author{
R.V. O'Toole III ${ }^{1,2}$, D.A. Simon ${ }^{2}$, \\ B. Jaramaz ${ }^{1,2}$, O. Ghattas ${ }^{3}$, M.K. Blackwell ${ }^{2}$, L. Kallivokas ${ }^{1}$, \\ F. Morgan ${ }^{2}$, C. Visnic ${ }^{1}$, A.M. DiGioia $\mathrm{III}^{1,2,3}$, and T. Kanade ${ }^{2}$ \\ 1 Center for Orthopaedic Research, Shadyside Hospital \\ 2 The Robotics Institute, Carnegie Mellon University \\ 3 Department of Civil Engineering, Carnegie Mellon University \\ Pittsburgh, PA
}

\begin{abstract}
Current surgical robotic systems in orthopaedics lack realistic pre-operative simulations and utilize invasive methods to register bone intra-operatively. A multidisciplinary group of researchers is addressing these deficiencies in the context of robotic cementless hip replacement surgery. In this paper we outline our current research progress and a road-map for the short-term future of our research agenda. This paper addresses four components of this effort: (1) realistic anatomical modeling, (2) biomechanics-based simulations, (3) surface-based registration, and (4) surgical robotics. We are integrating these components with the goal of developing more capable and less invasive robotic systems for use in orthopaedic surgery.
\end{abstract}

\section{Introduction}

The field of orthopaedics presents excellent opportunities for the incorporation of robotic and computer-based technologies to improve surgical techniques. Procedures such as total joint replacement are performed in large numbers and at significant cost each year. Over 300,000 total hip and knee replacements occur annually in the U.S. alone [3]. The short and long term clinical success of these surgical procedures depends strongly on the proper alignment, placement, and fit of the implant within the bony structure [9]. The clinical importance of precision and accuracy, along with the large number and high cost of the surgical procedures, indicates that important contributions can be made through the use of surgical robots and computer-based pre-operative planning and simulation in orthopaedics.

Figure 1 outlines the four basic components of our research effort: (1) realistic anatomical modeling, (2) biomechanics-based simulations, (3) surface-based registration, and (4) surgical robotics. We are integrating these components with the goal of developing more capable and less invasive robotic systems for use in orthopaedic surgery.

In biomechanics, our goal is to allow a surgeon to simulate the mechanical consequences of a proposed surgery, and to change surgical strategies based 
Fig. 1. Interaction of Research Topics

upon these consequences. By coupling this realistic simulation and planning capability with precise surgical robots, the surgeon can not only plan an "ideal" surgery, but also ensure that it is carried out. To execute a surgical plan with a robot, the system must possess the ability to register (determine the position and orientation) a bone in the clinical environment. Surface-based registration is desirable because it does not require fiducials to properly align the pre-operative plan with the patient's anatomy. The success of both surgical registration and pre-operative simulation is highly dependent upon the realism of geometric and physical models. As such, anatomic modeling has also emerged as a distinct research area. Our work attempts to join these four seemingly disparate research topics into one integrated effort to improve techniques in orthopaedic robotics.

\section{Modeling}

While algorithms exist for the creation of geometric models from volumetric medical data, little work has been published on the validation of these models. One reason for this lack of research is that until recently, geometric surface models have been used primarily for visualization tasks that do not demand highly accurate models. With the increased use of surface models for pre-operative planning and intra-operative guidance, geometric model accuracy has taken on new importance. Physical modeling issues subsume those associated with geometric modeling. In addition to difficulties in generating the geometry of the bone and implant, physical models must appropriately represent the underlying constitutive laws. 


\subsection{Geometric Model Creation and Validation}

The primary geometric model in our work is the polyhedral surface mesh. It is used for visualizing 3D surface geometries and for surface-based intra-surgical registration. A variety of techniques are available for reconstructing polyhedral surface meshes directly from CT or MRI data [6]. We currently generate surface meshes using several of these methods [2][8][1].

Geometric surface model validation is complicated since errors can be introduced at several stages of model creation: during imaging, segmentation, and surface creation. Furthermore, there are multiple measures of error that can be used (e.g. Hausdorff distances, difference volumes, surface normal differences). Since different tasks place different requirements upon the underlying model, validation criteria should be application dependent. For example, a geometric model used to specify pre-operative prosthesis placement may have very different accuracy requirements than one used for surface-based registration.

Geiger [2] provides an excellent discussion of surface model validation assuming idealized input data. He uses an analytical model of a torus instead of actual CT data to evaluate surfaces generated using several approaches. This work provides a first step towards the validation of surface models derived from clinically realistic CT data. The following list suggests a progression of experiments (in order of increasing complexity and realism) that could be performed to reach this goal:

1. Analytic model of a solid (cylinder, torus) - no CT data

2. CT images of physical objects that can be analytically modeled (cylinder, torus), constructed from bone analog

3. CT images of anatomical phantoms (femur, pelvis) made from bone analog

4. CT images of cadaver anatomy (femur, pelvis)

A fundamental issue in model validation is determining the "ground truth" to which the reconstructed model will be compared. Ground truth can either be determined by accurately sensing an existing object, or by accurately manufacturing an object based on an existing model. In Experiments 2 and 3, we can use either method for obtaining ground truth, whereas in Experiment 4 we must rely on accurate sensing.

An important measure of clinical realism in the above experiments is the ease with which an object of interest can be segmented from surrounding objects. In order to study this issue at early stages of experimentation, we are using CT soft tissue analogs. By surrounding an object with soft tissue analog during imaging, we complicate the segmentation process and make the resulting images closer to clinical reality. A second measure of experimental realism is the spatial density variation within an object. Density variations, like that in real bone, complicate segmentation by reducing the effectiveness of simple thresholding schemes. Cadaver studies allow us to study both of the above effects. In cadaver studies, bones would be imaged within the cadaver and then dissected out and used to build highly accurate ground truth models. We are currently investigating Experiment 2 and plan to progress to Experiments 3 and 4. 


\subsection{Physical Model Creation}

The geometric modeling of biological surfaces is a fairly well understood process. This is not the case for the accurate physical modeling of complex biological systems such as the bone-implant systems in total joint replacements. Fundamental research is still needed to create computer models that realistically mimic the biomechanical properties of the bone-implant construct.

In contrast to bone, physical modeling of an implant is relatively straightforward since the material properties are well known and a geometric description can be obtained from CAD models. The situation is much more difficult for a biological material such as bone. To simulate the mechanical consequences of a total hip replacement surgery, the bone model must accurately represent the geometric complexity and spatial distribution of material properties of a patient's femur and pelvis. Furthermore, bone exhibits a complex constitutive law with significant anisotropy, viscoelasticity, and nonlinearity. Additional nonlinearity arises from the contact between the implant and bone, with the interface depending on numerous geometric, material, and loading parameters.

When developing biomechanical models, there are a variety of options which progressively add realism to the resulting physical models. The types of models we are currently investigating fall into one of the following four broad categories:

1. Idealized (e.g axisymmetric) geometry, and complex material properties both based upon the literature.

2. Idealized geometry, and complex material properties — both derived directly from CT scan data.

3. Full 3D geometry based on CT data, but with idealized material properties.

4. Full 3D geometry, and complex material properties - both derived directly from CT scan data.

The categories range from least (1) to most (4) clinically realistic. There is a tradeoff between model complexity and the computational resources required to perform a simulation with a given model. The simplest model that accurately captures patient-specific mechanics is the best; however, more detailed models are still useful to help validate simplified approaches. As such, we are examining models that fall into all of the above categories.

Our initial approach was to develop simplified axisymmetric models to simulate the implantation of femoral and acetabular components (Category 1 above). These models incorporated idealized material properties with bi-linear elastic stress response and contact elements [10] [16]. While the bone geometries and material properties were not derived from CT data, these models incorporated many of the biomechanical complexities. Next, to help validate the results derived with the first set of models, full 3D irregular mesh FEM models were developed with geometry based upon CT scan data, but using material properties from the literature (Category 3 above).

Ideally, we wish to create realistic physical models directly from CT scans (Category 4). One option for such models is an irregular tetrahedral mesh that is grown directly from the CT data. Although potentially yielding a very realistic 
simulation, the disadvantage of this approach is the difficulty in growing and solving large irregular meshes. A second option is the use of a regular grid to represent the CT data, while embedding the unstructured implant model within the regular bone grid. Material properties can be based on the CT numbers using experimentally derived relations. The disadvantage of this approach is that it may yield a mesh that is too large unless the resolution is lowered. On the other hand, special fast algorithms that exploit the regular structure can be derived. Currently we are pursuing both the irregular and regular mesh options.

\section{Simulations}

Software systems exist that allow a surgeon to plan a surgery on the computer before entering the operating room. In orthopaedics, a clinical system now exists that can carry out a pre-operative plan precisely for the femoral part of total hip replacements [15]. In such systems, the surgeon can no longer rely on intra-operative feedback to determine the proper placement and fit of a pressfit implant. Instead, the surgeon must make these decisions by interacting with geometric implant models and the CT data to plan the surgery on a computer. By adding simulations of the mechanical consequences of a proposed surgery, it may be possible to compensate for the lack of intra-operative feedback in the current scenario.

We have simulated the press-fit insertion of cementless acetabular and femoral components for total hip replacements. Unlike previous efforts which have assumed an initial line-to-line fit between the implant and bone, our models and simulations have included contact coupling to simulate the actual implantation process [10] [16]. We argue that since the short term success of the implants depends strongly on the post-operative mechanical environment, this type of simulation provides valuable information in planning the surgery.

Currently, we are working towards simulations using more realistic models of the bone-implant system, as described above. These simulations will also incorporate contact coupling between bone and implant that occurs during the forceful insertion of the oversized implant into the bone cavity. The choice of model will dictate which solvers we ultimately use. For unstructured meshes we are developing preconditioned conjugate gradiant methods. We are also developing fast multigrid methods that exploit the structure of the regular meshes.

A surgical simulation must be realistic, but must also run fast enough that the information is useful clinically. Currently our analyses require between several hours (simple models), to several weeks (full 3D models), using commercial code and low-end workstations. By incorporating the latest algorithms and using highend workstations, we anticipate performing biomechanical simulations in near real-time.

Once realistic surgical simulations exist, work can progress on parametric studies of total joint replacement. Variables include the implant size and placement, and the shape of the bone cavity. The long-term goal of this work is not 
only to model the consequences of a surgery, but to create a system that can suggest optimal parameters to the surgeon.

\section{Registration}

Intra-surgical registration is the process of establishing a common reference frame between pre-surgical data and the corresponding patient anatomy. Once a common reference frame is established, pre-surgical data can be used to guide robotic tool movements [7], superimpose graphical overlays of internal anatomy upon a surgeon's view of the patient [4], position radiosurgical equipment [13], or guide a surgeon's tool movements [12]. Recent clinical approaches to intrasurgical registration assume a known correspondence between points in the two data sets being registered [15]. This is usually achieved by attaching fiducials to the underlying object, and extracting the locations of these markers in both data sets. Unfortunately, attachment of fiducials typically require an additional surgical procedure prior to the collection of pre-surgical data. Furthermore, these fiducials are invasive and cause added trauma to the patient in sites far from the primary surgical field.

An alternative to fiducial-based registration is to use surfaces that are intrinsic to the data itself. If data from the bounding surface of an object can be extracted pre- and intra-surgically, these data sets can be matched to perform registration. Several research groups have investigated such surface-based methods in medical registration [14][4][11][5]. A benefit of these techniques is that they do not require the use of costly and invasive external markers. Surfacebased methods, however, place a heavy burden on sensing and modeling technology since accurate pre- and intra-surgical surface data are needed. This is a much more difficult sensing task than acquiring $3 \mathrm{D}$ fiducial locations as required by previous approaches.

As we demonstrated in [14], the accuracy resulting from surface-based registration depends highly on the underlying data. These data include the geometric surface models from pre-operative CT scans, as well as data collected intra-surgically using digitizing probes, ultrasound, fluoroscopes or CT. We are currently developing methods for planning the acquisition of potentially costly intra-surgical registration data using pre-operative geometric models as input. The goal of this work is to select data that will result in the best possible registration accuracy, while minimizing the quantity of data required.

\section{Robotics}

In total hip replacement surgery, the modeling, simulation, and registration components manifest themselves in the robotic milling of a bone cavity. The focus of our work is on the milling of the acetabulum (socket in the pelvis) to prepare for the implantation of a cementless acetabular component. We have developed a robotic testbed to demonstrate registration and cutting strategies. The testbed is not designed for clinical use, so issues of sterility, sensor redundancy, and 
clinical safety have not been addressed. Our system consists of a 5 DOF SCARA direct drive manipulator (Adept, San Jose, CA) with an attached 6 axis forcetorque sensor (JR3 Inc, Woodland, CA) and pneumatic milling tool. The overall system configuration is conceptually similar to that used by Taylor [15].

A pre-operative plan is defined using our custom planner and models of the implants. There are three reference frames of interest: (1) the CT reference frame (where the pre-operative plan is defined), (2) the intra-operative sensor reference frame (where bone surface data are defined), and (3) the robot reference frame (where the actual robot paths are defined). Intra-operative surface data and a surface model from the CT data are used to determine the transformation between sensor and CT reference frames (as described in Section 4). The transformation between the sensor and the robot frames is derived by repeatedly moving the robot and sensor to the same point in space, recording the locations, and then calculating an average transformation matrix.

The above process yields the pose of the bone and a cutting plan in the robot's coordinates. Shapes in the bone can be milled by first making broad cuts to remove large amounts of material in a short time, and then returning to finish the cavity with finer passes. We have demonstrated (on pelvis bone phantoms) milling of hemispherical cavities that match the shape of the implants, but are undersized to allow varying amounts of press-fit. These shapes are high precision versions of the cavities created with standard hand-held tools, but arbitrary shapes could be generated should the biomechanics simulations predict that a non-hemispherical cavity is preferable.

Current work centers on milling less-rigidly fixed bones. With high speed tracking it may be possible to do away with rigid fixation and allow some small (millimeters) motion of the bone. The robotic system would compensate for this motion. The accuracy and bandwidth requirements of such a system are strenuous, and much work is needed to evaluate the clinical efficacy of the concept.

\section{Conclusion}

The value of surgical robots is greatly enhanced by realistic surgical simulations, less invasive registration methods, and accurate modeling. Surgical simulations will enhance the surgeon's ability to investigate the implications of pre-operative plans. Surface-based registration will eliminate invasive and costly fiducials which limit the clinical application of orthopaedic robotics. Accurate modeling is a prerequisite for realistic simulations and precise intra-operative registration. Each of these components are worthy research efforts on their own; together, they form a framework for advancing the clinical utility of medical robotics.

\section{References}

1. H.N. Christiansen and T.W. Sederberg. Conversion of complex contour line definitions into polygonal element mosaics. Computer Graphics, 8:658-660, August 1978. 
2. B. Geiger. Three-dimensional modeling of human organs and its application to diagnosis and surgical planning. PhD thesis, Ecole des Mines de Paris, April 1993.

3. E.J. Graves. 1992 summary: National hospital discharge survey. In Advance Data from Vital Health and Statistics, page 45. National Center for Health Statistics, 1992.

4. E Grimson, T. Lozano-Perez, W. Wells, G. Ettinger, S. White, and R. Kikinis. Automated registration for enhanced reality visualization in surgery. In $A A A I$ 1994 Spring Symposium Series, Applications of Computer Vision in Medical Image Processing, pages 26-29. AAAI, March 1994.

5. H. Jiang, R.A. Robb, and K.S. Holton. A new approach to 3-d registration of multimodality medical images by surface matching. In Visualization in Biomedical Computing - SPIE Vol 1808, pages 196-213. SPIE, 1992.

6. A.D. Kalvin. A survey of algorithms for constructing surfaces from 3D volume data. IBM Reseach Report RC 17600, IBM, January 1992.

7. J.T. Lea, D. Watkins, A. Mills, M.A. Preshkin, T.C. Kienzle, and S.D Stulberg. Registration and immobilization for robot-assisted orthopaedic surgery. In Proceedings of the First International Symposium on Medical Robotics and Computer Assisted Surgery, pages 63-68, Pittsburgh, PA, September 1994.

8. W.E. Lorensen and H.E. Cline. Marching cubes: A high resolution 3D surface construction algorithm. Computer Graphics, 21:163-169, July 1987.

9. V.C. Mow and W.C. Hayes. Basic Orthopaedic Biomechanics. Raven Press, 1991.

10. R.V. O’Toole, B. Jaramaz, A.M. DiGioia, C.D. Visnic, and R.H. Reid. Biomechanics for pre-operative planning and surgical simulation in orthopaedics. Computers in Biology and Medicine, 1994.

11. O. Peria, A. Francois-Joubert, S. Lavallee, G. Champleboux, P. Cinquin, and S. Grand. Accurate registration of SPECT and MR brain images of patients suffering from epilepsy or tumor. In Proceedings of the First International Symposium on Medical Robotics and Computer Assisted Surgery, pages 58-62, Pittsburgh, PA, September 1994.

12. K. Radermacher, H.W. Staudte, and G. Rau. Computer assisted orthopedic surgery by means of individual templates - aspects and analysis of potential applications. In Proceedings of the First International Symposium on Medical Robotics and Computer Assisted Surgery, pages 42-48, Pittsburgh, PA, September 1994.

13. A. Schweikard, R. Tombropoulos, J.R. Adler, and J. Latombe. Planning for imageguided radiosurgery. In AAAI 1994 Spring Symposium Series, Applications of Computer Vision in Medical Image Processing, pages 96-101. AAAI, March 1994.

14. D.A. Simon, M. Hebert, and T. Kanade. Techniques for fast and accurate intrasurgical registration. In Proceedings of the First International Symposium on Medical Robotics and Computer Assisted Surgery, pages 90-97, Pittsburgh, PA, September 1994.

15. R.H. Taylor, B.D. Mittelstadt, H.A. Paul, W. Hanson, P. Kazanzides, J.F. Zuhars, B Williamson, B.L. Musits, E. Glassman, and W.L. Bargar. An image-directed robotic system for precise orthopaedic surgery. IEEE Transactions on Robotics and Automation, 10(3):261-275, June 1994.

16. C.D. Visnic, R.H. Reid, A.M. DiGioia, B. Jaramaz, and O. Ghattas. Finite element pre-operative simulation of cementless hip replacement. In Proceedings of the 1994 Winter Simulation Conference, 1994. 